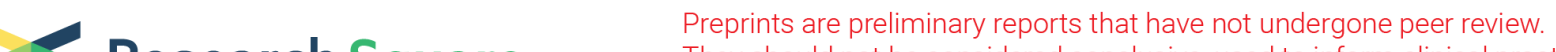 Research Square They should not be considered conclusive, used to inform clinical practice, or referenced by the media as validated information.
}

\section{The use of Fretsaw as a Substitute Osteotomy Tool while Oscillating Saw Malfunction in Hip Arthroplasty}

\section{Zanjing Zhai}

Shanghai 9th Peoples Hospital Affiliated to Shanghai Jiaotong University School of Medicine

\section{Yongyun Chang}

Shanghai 9th Peoples Hospital Affiliated to Shanghai Jiaotong University School of Medicine https://orcid.org/0000-0001-7191-9573

\section{Degang Yu}

Shanghai 9th Peoples Hospital Affiliated to Shanghai Jiaotong University School of Medicine

\section{Huiwu Li}

Shanghai 9th Peoples Hospital Affiliated to Shanghai Jiaotong University School of Medicine

\section{Yuanqing Mao}

Shanghai 9th Peoples Hospital Affiliated to Shanghai Jiaotong University School of Medicine

\section{Yiming Zeng}

Shanghai 9th Peoples Hospital Affiliated to Shanghai Jiaotong University School of Medicine

\section{Mengning Yan ( $\square$ mengningyan@163.com )}

Shanghai Jiao Tong University School of Medicine https://orcid.org/0000-0002-2381-0028

\section{Research article}

Keywords: Total hip arthroplasty, femoral neck osteotomy, oscillating saw, fretsaw

Posted Date: February 5th, 2021

DOI: https://doi.org/10.21203/rs.3.rs-160934/v1

License: (c) (1) This work is licensed under a Creative Commons Attribution 4.0 International License. Read Full License 


\section{Abstract}

Background: The occurrence of oscillating saw malfunction, power shortage, or contamination occurs frequently when implementing femoral neck osteotomy during total hip arthroplasty (THA). This study aimed to introduce the fretsaw as a novel substitute osteotomy tool with various advantages.

Methods: Twenty patients (20 hips) who underwent primary THA were included. Ten patients underwent femoral neck osteotomy using a fretsaw, while the other 10 patients underwent the procedure using an oscillating saw. Intraoperative evaluation and radiographic data were obtained for all patients during and after surgery.

Results: The mean osteotomy time was $20.60 \pm 1.08$ s (range 16-27) and $22.10 \pm 1.49$ s (range 16-31) in the oscillating saw and fretsaw groups, respectively. The mean osteotomy height was $1.21 \pm 0.16$ (range $1.01-1.43) \mathrm{cm}$ and $1.14 \pm 0.08$ (range 1.02-1.28) $\mathrm{cm}$ in the oscillating saw and fretsaw groups, respectively. The use of fretsaw did not result in bone notch or blood splashes.

Conclusion: The fretsaw can be a substitute femoral neck osteotomy tool with various advantages in THA while oscillating saw malfunction.

\section{Background}

Total hip arthroplasty (THA) is a frequently performed and highly successful surgery for patients with end-stage hip disease. It can relieve pain, enhance function, and improve patient quality of life[1, 2]. Globally, more than one million THAs are performed each year, and the number has increased annually[3]. Since the pioneering work of Wiles, Charnley, and others in the mid-20th century, implant technology and instruments have steadily been improved and perfected[4]. Nowadays, the procedures and devices of THA have become normalized and routinized. However, complications still occasionally occur due to faults with the instruments.

Problems associated with the oscillating saw include power shortage and contamination when performing femoral neck osteotomy during THA. Presently, there is a lack of a suitable alternative osteotomy tool. Moreover, the oscillating saw has certain disadvantages of its own (Fig. 1). Firstly, the oscillating saw tends to sway due to the strong reverse impact when the saw touches bone, potentially resulting in cortical bone injury[5]. Secondly, notch formation can occur on the osteotomy position. The oscillating saw may also lead to the incidence of soft tissue and greater trochanter damage[6] (Fig. 1BD). Furthermore, the oscillating saw generates a high frequency of blood splash during surgery[7], which puts surgeons at greater risk of disease transmission (Fig. 1A, 1E). Lastly, due to the dead zone at the contralateral side of the femoral neck, it is difficult to estimate the osteotomy border, which increases the risk of damage to the soft tissue and acetabular by the oscillating saw.

In our previous clinical work for decades, we identified the fretsaw could be an ideal substitute tool for femoral neck osteotomy when oscillating saw malfunction during THA. Moreover, using a fretsaw has no 
risk of notch formation, decreases the risk of soft tissue and greater trochanter damage, and does not generate blood splash. This study aimed to describe the use of fretsaw as a substitute osteotomy tool in THA, especially when oscillating saw malfunction.

\section{Methods}

The study was conducted in accordance with the Declaration of Helsinki. The study was approved by Ethics Committee of Shanghai Ninth People's Hospital affiliated to Shanghai Jiao Tong University School of Medicine and informed consent was taken from all individual participants.

\section{Patients}

Twenty patients (20 hips) were evenly randomly divided into two groups. Patients eligible for inclusion were those undergoing primary THA due to femoral neck fracture, femoral head necrosis, developmental hip dysplasia (Crowe I), or primary osteoarthritis using a posterolateral approach and a cementless pressfit cup. The exclusion criteria were as follows: those with severe deformity of the femoral neck or hip ankylosis. In the fretsaw group, 10 patients underwent femoral neck osteotomy with fretsaw (5 males and 5 females, $66.3 \pm 16.1 \mathrm{ys}$ ), while the other 10 patients, in the oscillating saw group, underwent femoral neck osteotomy with oscillating saw ( 5 males and 5 females, $63.4 \pm 19.8$ ys).

\section{Surgical technique}

All operations were performed by the same group of surgeons using a posterolateral approach under general anesthesia. The femoral neck was osteotomized using either a fretsaw or oscillating saw (Fig. 2 and supplemental video). The remaining procedures were identical between the two groups.

\section{Evaluation method}

Clinical and radiographic data were obtained for all patients during and after surgery. Intraoperative evaluation, including osteotomy time (calculating the time between the beginning of osteotomy, with either a fretsaw or oscillating saw, and the femoral neck division), osteotomy height (the heights of the osteotomies were measured from the lesser trochanter to the cuneiform plane), notch formation, and blood splash generation (calculating the surgical masks with blood splashes after surgery). Standard radiographs included a routine anteroposterior (AP) view of the pelvis and proximal femur.

\section{Statistical analysis}

SPSS software (Version 19; SPSS Inc., Chicago, IL) was used for statistical analysis. Student's t-test was used to compare means of the clinical results. A P value less than 0.05 was considered to be significant.

\section{Results}


The femoral neck osteotomy time is shown in Fig. 3A. The mean osteotomy time ( \pm standard deviation, SD) for the oscillating saw and fretsaw groups was $20.60 \pm 1.08 \mathrm{~s}$ (range 16-27 s) and $22.10 \pm 1.49 \mathrm{~s}$ (range 16-31 s), respectively. There was no significant difference in osteotomy time between the two groups $(P>0.05)$.

The femoral neck osteotomy height is shown in Fig. 3B. The mean osteotomy height for the oscillating saw and fretsaw groups was $1.21 \pm 0.16 \mathrm{~cm}$ (range $1.01-1.43 \mathrm{~cm}$ ) and $1.14 \pm 0.08 \mathrm{~cm}$ (range 1.02-1.28 $\mathrm{cm})$, respectively. There was no significant difference in osteotomy height between the two groups $(P>$ 0.05 ). All procedures fell within the target osteotomy height of $1.0-1.5 \mathrm{~cm}$.

There are four bone notches (4 hips) formed in oscillating saw group, while none in fretsaw group (Fig. 3C). In the fretsaw group, no blood splash was generated during the femoral neck osteotomy. However, many splashes were generated when using the oscillating saw, landing on the mask, glasses, and forehead of surgeons and assistants. In our study, there are four doctors in each operation. Statistically, twenty-six surgical masks with blood splashes occur in oscillating saw group while none in fretsaw group (Fig. 3D). The intra-operative process was smooth and postoperative radiographs showed no prostheses malposition in both fretsaw and oscillating saw groups (Fig. 4).

\section{Discussion}

Malfunction, power shortage, or contamination of oscillating saw, as a femoral neck osteotomy tool in THA, could happened from time to time. Re-sterilization or replacement of the instruments adds to the cost of providing the instruments and also be time consuming[8]. To the best of our knowledge, this is the first study that introduce the fretsaw as a substitute osteotomy tool in THA. This study demonstrates the satisfactory osteotomy outcomes, as well as distinct advantages, of using the fretsaw for this purpose.

Operation time is an important factor in intraoperative safety and postoperative rehabilitation of patients[9]. In the present study, there was no significant difference in osteotomy time between the fretsaw and oscillating saw groups. The fretsaw was equally efficient as a tool for femoral neck osteotomy and did not prolong the operative time. In our study, the fretsaw group achieved a satisfactory osteotomy height, which was consistent with preoperative planning. There was no significant difference in osteotomy height between the two groups. More interestingly, the deviation of the fretsaw group was lower than that of the oscillating saw group. This might be related to the saw blade swaying due to the strong reverse impact when the high-speed saw blade touches bone.

Notch generation in the femoral neck contributes to a high stress concentration during canal preparation and stem implantation, increasing the risk of Intraoperative periprosthetic femoral fracture (IOPFF)[1012]. In our study, using a fretsaw as the femoral neck osteotomy tool resulted in a smooth osteotomy plane, and there is no occurrence of notch generation. 
Blood splash occurs frequently when using an oscillating saw for femoral neck osteotomy. This puts surgeons and assistants at greater risk of infection with blood-borne diseases transmitted from the patient[7]. Furthermore, patients are also susceptible to infection due to reverse splashes, which can deflect off a surface and contaminate the surgical site[13, 14]. A surgical mask cannot provide sufficient protection against blood splash. Many surgeons neglect eye and whole face protection due to discomfort or a misty field of view[15]. In our study, no blood splash was generated when using the fretsaw as the femoral neck osteotomy tool, demonstrating another advantage to using the fretsaw for this purpose.

Our study has several limitations. Firstly, the sample size of study group was relatively small. Secondly, the fretsaw was only used as the osteotomy tool in patients with a relatively normal femoral neck, such as those with osteonecrosis of the femoral head, femoral neck fracture, hip osteoarthritis, or dysplasia of the hip (Crowe type I). Patients with more severe deformity of the femoral neck or hip ankyloses were not included.

\section{Conclusion}

This study demonstrated that the fretsaw was an effective femoral neck osteotomy tool with a number of advantages. While oscillating saw malfunction, power shortage, or contamination occurs in THA, the fretsaw could be an ideal substitute osteotomy tool.

\section{Abbreviations}

THA: Total hip arthroplasty; AP: Anteroposterior; SD: Standard deviation; IOPFF: Intraoperative periprosthetic femoral fracture

\section{Declarations}

\section{Authors' contributions}

Yiming Zeng and Mengning Yan conceived and designed the experiment. Zanjing Zhai and Yongyun Chang performed the experiment. Zanjing Zhai, Yongyun Chang and Degang Yu analyzed and interpreted the data. Huiwu Li and Yuanqing Mao supervised the study and provided administrative support. Zanjing Zhai and Yongyun Chang wrote the paper. All authors read and approved the final manuscript.

\section{Competing interests}

The authors have no conflicts of interest to declare.

\section{Funding}


This study was supported by the Science \& Technology Innovation Fund of Shanghai Ninth People's Hospital, Shanghai Jiaotong University School of Medicine (CK2019002); Shanghai Clinical Medical Center (2017ZZ01023); Shanghai Municipal Key Clinical Specialty (shslczdzk00402, shslczdzk07001); The Youth Doctor Collaborative Innovation Team Project of Shanghai Ninth People's Hospital, Shanghai Jiaotong University School of Medicine (QC201903).

\section{Availability of data and materials}

The datasets generated and analyzed during the current study are available from the corresponding author on reasonable request.

\section{Ethics approval and consent to participate}

The study was conducted in accordance with the Declaration of Helsinki. The study was approved by Ethics Committee of Shanghai Ninth People's Hospital affiliated to Shanghai Jiao Tong University School of Medicine and informed consent was taken from all individual participants.

\section{Consent for publication}

Written informed consent was obtained from all participants.

\section{Acknowledgements}

Not applicable.

\section{References}

1. Mellon SJ, Liddle AD, Pandit H. Hip replacement: landmark surgery in modern medical history. Maturitas 75(3): 221, 2013

2. Branson JJ, Goldstein WM. Primary total hip arthroplasty. AORN journal 78(6): 946, 2003

3. Ferguson RJ, Palmer AJ, Taylor A, Porter ML, Malchau H, Glyn-Jones S. Hip replacement. Lancet 392(10158): 1662, 2018

4. Learmonth ID, Young C, Rorabeck C. The operation of the century: total hip replacement. Lancet 370(9597): 1508, 2007

5. Dimitris K, Taylor BC, Steensen RN. Excursion of oscillating saw blades in total knee arthroplasty. J Arthroplasty 25(1): 158, 2010

6. Herregodts S, Verhaeghe M, Paridaens R, Herregodts J, Vermue H, Arnout N, De Baets P, Victor J. Softtissue penetration of the oscillating saw during tibial resection in total knee arthroplasty. Bone Joint 
J 102-B(10): 1324, 2020

7. Alani A, Modi C, Almedghio S, Mackie I. The risks of splash injury when using power tools during orthopaedic surgery: a prospective study. Acta Orthopædica Belgica 74(5): 678, 2008

8. Cichos KH, Hyde ZB, Mabry SE, Ghanem ES, Brabston EW, Hayes LW, McGwin G, Jr., Ponce BA. Optimization of Orthopedic Surgical Instrument Trays: Lean Principles to Reduce Fixed Operating Room Expenses. J Arthroplasty 34(12): 2834, 2019

9. Krebs VE, Elmallah RK, Khlopas A, Chughtai M, Bonutti PM, Roche M, Mont MA. Wound Closure Techniques for Total Knee Arthroplasty: An Evidence-Based Review of the Literature. J Arthroplasty 33(2): 633, 2018

10. Lamb JN, Matharu GS, Redmond A, Judge A, West RM, Pandit HG. Patient and implant survival following intraoperative periprosthetic femoral fractures during primary total hip arthroplasty: an analysis from the national joint registry for England, Wales, Northern Ireland and the Isle of Man. Bone Joint J 101-B(10): 1199, 2019

11. Lamb JN, Matharu GS, Redmond A, Judge A, West RM, Pandit HG. Risk Factors for Intraoperative Periprosthetic Femoral Fractures During Primary Total Hip Arthroplasty. An Analysis From the National Joint Registry for England and Wales and the Isle of Man. J Arthroplasty 34(12): 3065, 2019

12. Ferbert T, Jaber A, Gress N, Schmidmaier G, Gotterbarm T, Merle C. Impact of intraoperative femoral fractures in primary hip arthroplasty: a comparative study with a mid-term follow-up. Hip Int 30(5): 544,2020

13. Singh VK, Kalairajah Y. Splash in elective primary knee and hip replacement: are we adequately protected? J Bone Joint Surg Br 91(8): 1074, 2009

14. Zhiqing L, Yongyun C, Wenxiang C, Mengning Y, Yuanqing M, Zhenan Z, Haishan W, Jie Z, Kerong D, Huiwu L. Surgical masks as source of bacterial contamination during operative procedures. Journal of orthopaedic translation 14: 57, 2018

15. Hirpara KM, O'Halloran E, O'Sullivan M. A quantitative assessment of facial protection systems in elective hip arthroplasty. Acta Orthop Belg 77(3): 375, 2011

\section{Figures}



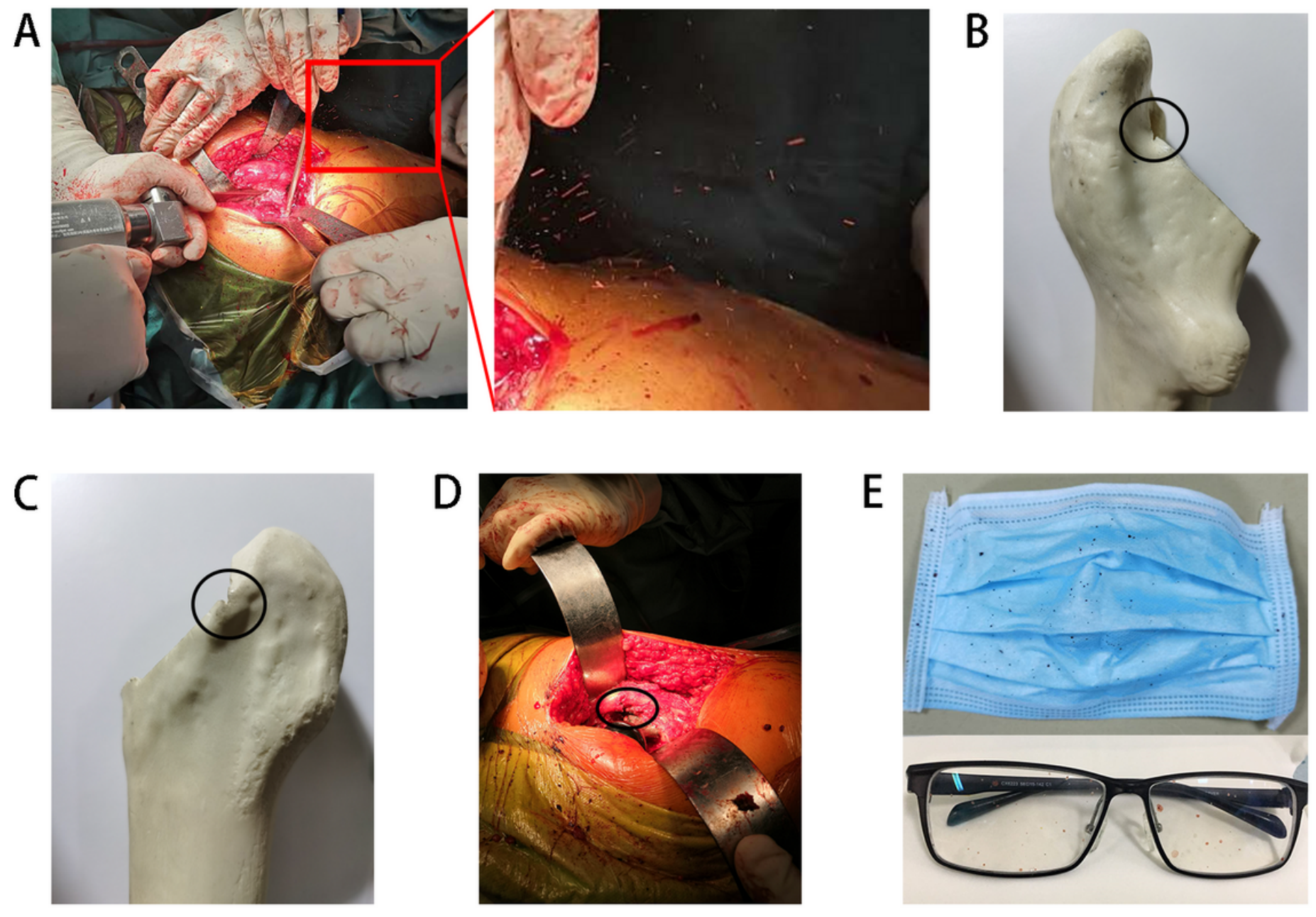

\section{Figure 1}

The deficiencies of the oscillating saw. Femoral neck osteotomy using oscillating saw during surgery and plenty of splashes generating (A). A notch on the intersecting position of the osteotomy ( $B, C)$. Damage to the soft tissue and greater trochanter as a result of the oscillating saw (D). Blood splashes generated by the use of oscillating saw on the masks and glasses (E). 

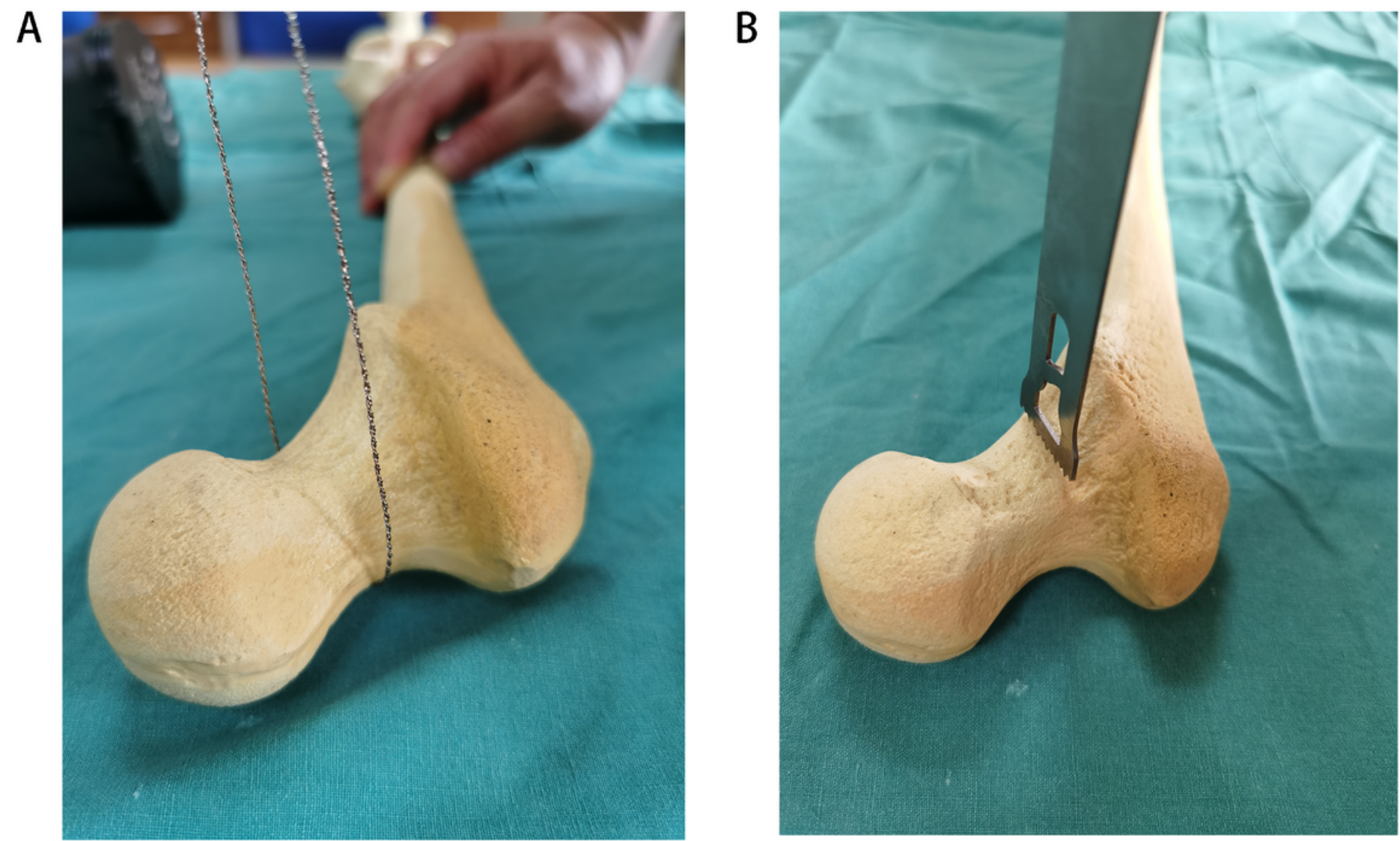

Figure 2

Photograph of a fretsaw (A) and an oscillating saw (B) as femoral neck osteotomy tool. 
A

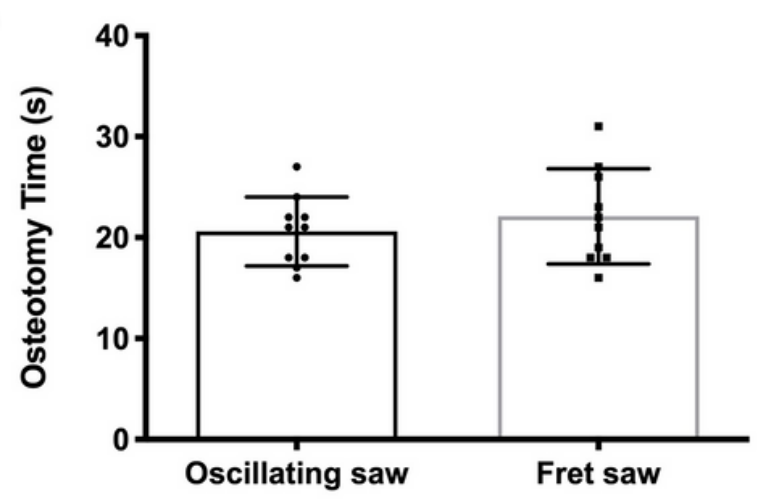

C

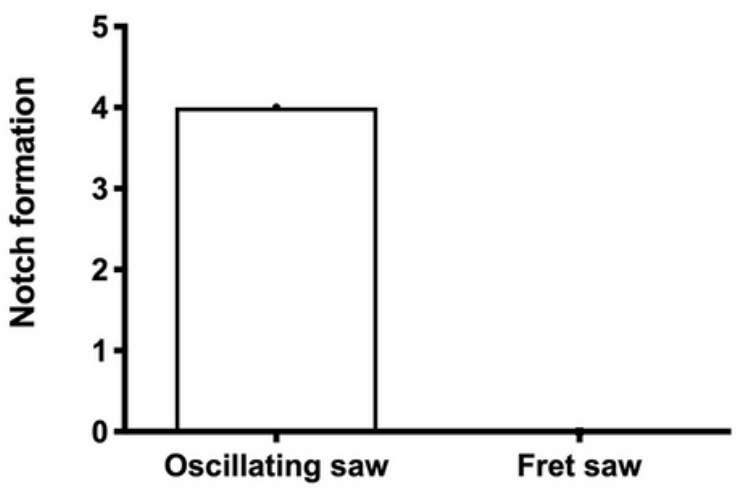

B

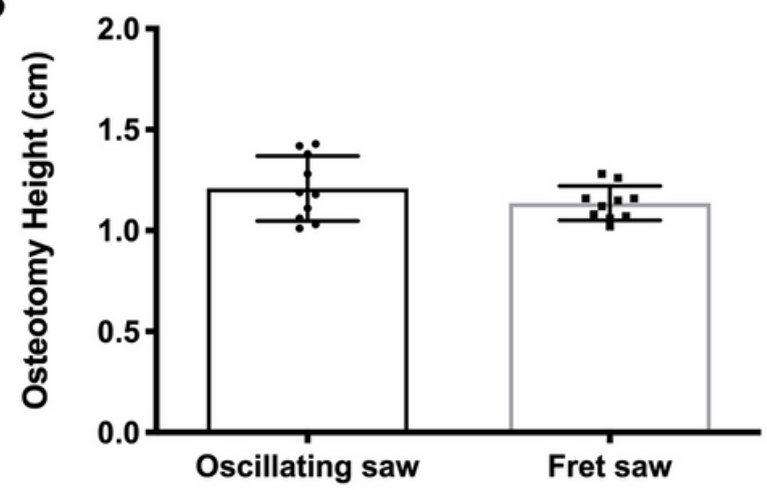

D

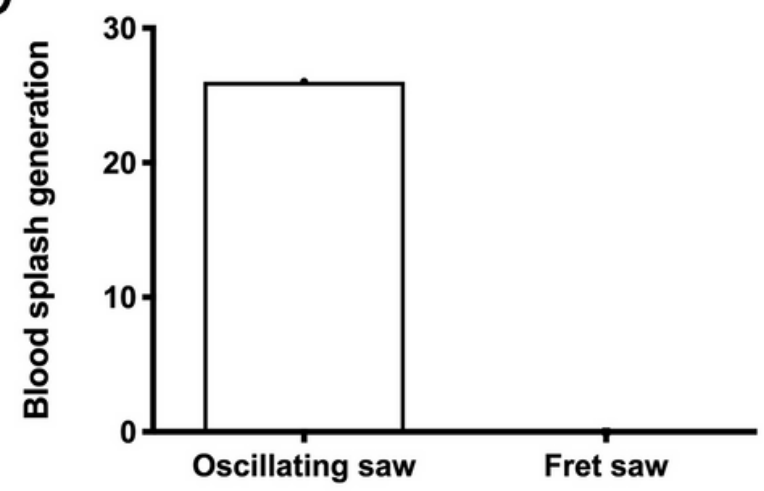

Figure 3

The osteotomy time (A) and height (B) when using the fretsaw and oscillating saw. There are four notches (4 hips) formed in oscillating saw group, while none in fretsaw group (C). Twenty-six surgical masks with blood splashes occur in oscillating saw group while none in fretsaw group (D). 

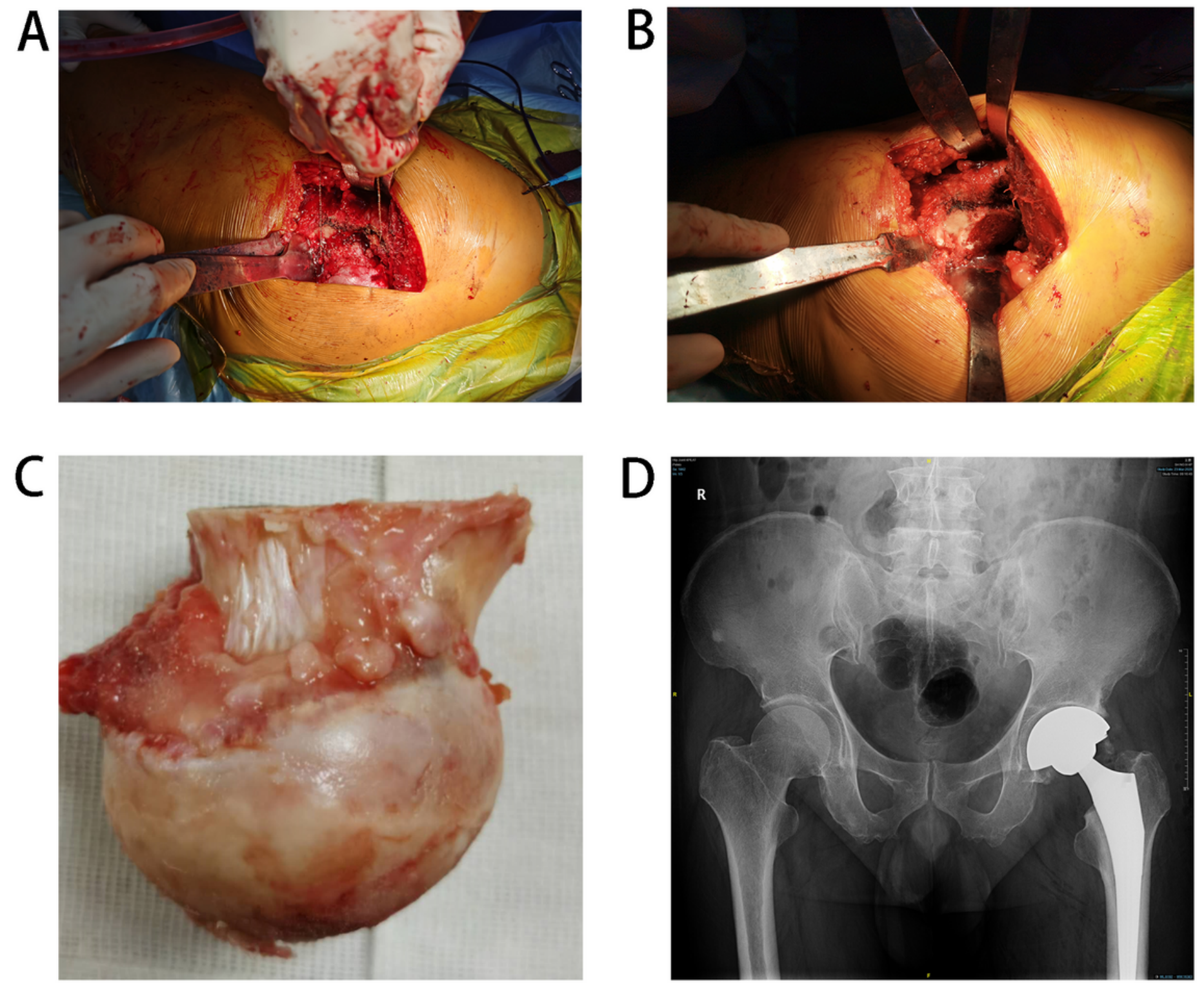

\section{Figure 4}

Intraoperative photographs and radiographic data were obtained for all patients during and after surgery. Osteotomy using a fretsaw (A). Intraoperative photographs of the fretsaw group (B, C). Radiographs of a patient within the fretsaw group after surgery (D).

\section{Supplementary Files}

This is a list of supplementary files associated with this preprint. Click to download.

- supplementalfigure.mp4.mp4 